\title{
Immer wieder „Trauma“?
}

\section{Ulrich Lamparter}

Online publiziert: 10. August 2011

(C) Springer-Verlag 2011

Diese Ausgabe des Forum der Psychoanalyse ist dem Trauma gewidmet. Muss das sein? Gibt es nicht genug hervorragende Literatur zu diesem Thema? Und schon lange!

In der Tat - untrennbar ist die Geschichte der Erforschung der Folgen seelischer Verletzungen mit der Geschichte der Psychoanalyse verbunden, und entsprechend lang ist die Geschichte der Beschäftigung mit dem Trauma: Mit Sigmund Freud ist die Frage nach der traumatischen Ätiologie der Neurosen zu einer zentralen heuristischen Frage geworden. Sie wirft Licht auf die Mechanismen der innerseelischen Prozesse und hilft damit, die Subjektivität des Menschen wissenschaftlich zu ergründen. Mag der Traum die Via regia zum Unbewussten gewesen sein; das Trauma erweist sich immer wieder als die Via regia zur Klärung der Beziehung zwischen Innenwelt und Außenwelt. Hier stehen die im intimen Dialog in der Psychoanalyse gewonnenen Erkenntnisse für beides: einerseits die langfristigen Folgen einer erlittenen seelischen Verletzung und andererseits die zwangsläufigen Zusammenhänge ihrer Auswirkung mit den innerseelischen Kräften, unbewussten Motiven und Bedeutungen.

Nicht immer gelang es in der Geschichte der Psychoanalyse und ihrer Behandlungspraxis, angemessen mit dem Trauma umzugehen. Bereits Freud sah sich ja zur Aufgabe der „Verführungstheorie“ genötigt und gelangte zur Entdeckung der ödipalen Strukturen. In dieser Spannung hat die psychoanalytische Terminologie ein Begriffsrepertoire entwickelt, das die Vielgestaltigkeit relevanter Prozesse abzubilden vermag und das enorm hilfreich ist. Schon allein der kürzlich von Nedelmann (2011) in den Fokus gerichtete Blick auf die vielfältigen Zusammenhänge von Fantasie und Realität kann zum Beispiel bei der Erfassung und Beschreibung eines traumatischen Geschehens und seiner Folgen eine optimale Einstellung vermitteln.

PD Dr. med. Dipl.-Psych. U. Lamparter ( $ه)$

Institut und Poliklinik für Psychosomatische Medizin und Psychotherapie,

Universitätsklinikum Hamburg-Eppendorf, Martinistr. 52,

20246 Hamburg, Deutschland

E-Mail: ulamparter@t-online.de 
Denn das Trauma ist immer beides: Einbruch einer Realität, die zunächst eben keine genuin psychische ist, und die unausweichliche psychische Verarbeitung dieses Traumas, selbst wenn diese nicht gelingt. Trauma wird so vom isolierten Ereignis zum Geschehen, das an unbewusste Prozesse angeschlossen ist und Abwehrvorgängen unterliegt.

Für die therapeutische Praxis heißt dies ebenso beides: einmal das Trauma und seine - manchmal scheinbar jäh abbrechende - Erinnerungsspur genau zu erkunden und die verletzten innerseelischen Strukturen zu ermitteln und zu versorgen, zum anderen ebenso genau und detailliert den Bedeutungsspuren eines Traumas und den damit verbundenen Abwehrprozessen nachzugehen. Diese Grundprinzipien der Behandlung des psychischen Traumas sind in der Psychoanalyse klar und zeitbeständig entfaltet. Dennoch gibt es viele Fragen, die immer wieder neu geklärt werden müssen:

Wo endet der traumatische Zusammenhang und wo beginnt die „subjektive Bedeutungsentnahme"? Wie sehr können wir uns auf die Erinnerung des Patienten verlassen? Wie ist die Übertragung bei traumatisierten Patienten zu verstehen und zu handhaben?

Wie kann eine sekundäre Traumatisierung in der Therapie verhindert werden?

Ist es richtig, dass jede Grenzverletzung traumatisch ist? Erfordern traumatische Strukturverletzungen eine besondere Behandlungstechnik? Wie können wir sicherstellen, nicht unbewusst einer gesellschaftlichen Abwehr von Traumatisierung zu unterliegen? Wie kann es uns gelingen, in einer allgemeinen Atmosphäre der medialen Skandalisierung von Trauma und Traumatisierung und ihrer gleichzeitigen Verharmlosung und Verleugnung unsere gedanklichen Werkzeuge sauber zu halten?

Die im Heft abgedruckten Arbeiten berichten aus diesem Spannungsfeld.

Die Arbeit von Klaus Wackernagel legt fundiert die aktuellen traumatheoretischen Positionen der Psychoanalyse dar und gibt gleichzeitig einen beeindruckenden Einblick in die psychoanalytische Langzeitbehandlung von schwer traumatisierten Patienten. Er vertritt eine eher konservative Position, die Arbeit am Trauma in die klassische Arbeit zu integrieren. Mit der Arbeit von Andrea Eckert wird eine manchen psychoanalytischen Denktraditionen gegenüber kritische Arbeit gegenübergestellt. Sie plädiert für eine etwas andere Art der Behandlungspraxis, die noch expliziter mit dem Nacherleben des Traumas arbeitet.

Bei der Lektüre beider Arbeiten wird das häufig gehörte Vorurteil, die Psychoanalyse beschäftige sich nur mit leicht kranken Patienten, obsolet.

Die Arbeit von Franziska Lamott und Günter Lempa verweist auf Fragestellungen, die sich aus der Aktualität des Afghanistankriegs ergeben und wirft einen diskursanalytischen Blick auf die posttraumatische Belastungsstörung. Die ausschließlich auf bestimmte deskriptive Phänomene bezogene diagnostische Kategorie grenzt den psychotraumatischen Zusammenhang unzulässig ein. Traumafolgen sind mehr, möchte man laut rufen und als ersten klassischen Beleg neben den dissoziativen Symptomen und den entsprechenden Störungsbildern die Traumareaktivierung im Alter heranziehen. Die Autoren unterstützen die Stimmen, die schon lange vor einem unreflek- 
tierten Gebrauch der ICD ${ }^{1}$-Diagnosen warnen, indem sie auf die vorwiegende ökonomische Funktionalität dieser Klassifikationen hinweisen.

Sie zeigen im historischen Vergleich die gedanklichen Kontinuitäten auf, die kriegsbedingte Traumafolgen der individuellen Verletzlichkeit des Beschädigten anlasten. Diese Kontinuität reicht bis weit in die Auseinandersetzungen um die Kriegsbeschädigten des Ersten Weltkriegs zurück. Wieder einmal scheinen in einer kollektiven Verblendung und einer gesellschaftlichen Abwehr unangenehmer und belastender Wirklichkeiten und Wahrheiten das Ausmaß und die gesellschaftlichen Kosten einer Traumatisierung durch Kriege deutlich unterschätzt. Die scharfsinnige und kenntnisreiche Analyse der Autoren zeigt beispielhaft, in welcher Weise heute Bedarf an aufgeklärter Psychoanalyse besteht, einer Psychoanalyse, die gesellschaftliche Entwicklungen nicht nur als gegeben hinnimmt, sondern in der Lage ist, diese in einer Parteinahme für das Individuum kritisch zu beleuchten.

Auch diese Fähigkeit musste sich die Psychoanalyse in ihrer Geschichte hart und leidvoll erarbeiten und gerade die frühen Versuche, kriegstraumatisch bedingte Erkrankungen psychoanalytisch zu behandeln, können nur als erste Schritte auf diesem Weg gelten.

\section{Wo gibt es Schwachpunkte, wo gibt es Bedarf?}

In meiner Praxis sehe ich immer wieder an Gutachten, wie wenig der psychoanalytische Gedanke in der versorgenden Medizin und der konkreten Entschädigungspraxis entwickelt ist und wie sehr es nötig wäre, den Gerichten, Versicherungsträgern, Krankenkassen und Therapeuten in diesem Spannungsfeld mehr Orientierungen aus psychoanalytischer Sicht zu geben.

Mir selbst geht es manchmal so, dass ich etwas neidvoll auf die pragmatische Klarheit der Unfallchirurgen blicke. Diese betrachten die Geschichte, wie es zur Traumatisierung gekommen ist, eher als randständig und konzentrieren sich diagnostisch und therapeutisch auf die verletzten Strukturen. Man unterscheidet zwar auch zwischen der Art der erlittenen Einwirkung von außen (zum Beispiel stumpfes Bauchtrauma, Skiunfall), doch die therapeutische Strategie gibt die erlittene Verletzung (zum Beispiel Milzruptur, Sprunggelenkfraktur) vor. Diese Trennung scheint mir in der psychologischen Fachliteratur nicht immer versucht. Wir unterscheiden vielleicht zwischen schweren Traumen und leichteren, anhaltenden und eher kurzfristigen oder zum Beispiel Missbrauch, Unfall, häufiges Schlagen, sprechen vielleicht diffus sogar von ,traumatischen Erfahrungen und Erlebnissen“, haben jedoch nur wenige Begriffe für die Beschreibung der verletzten Strukturen zur Verfügung, unterscheiden gleichsam nicht systematisch die Art und Lage der Fraktur oder die Art der Verletzung der inneren Organe. Insofern wird vielleicht manche behandlungstechnische Debatte von völlig verschiedenen Vorstellungen aus geführt. Der zugegeben etwas medikozentristisch anmutende Vergleich mit der Unfallchirurgie zeigt ebenso anschaulich, dass es Unfälle gibt, bei denen ein Mensch wie von einem Schutzengel bewahrt aus den Trümmern seines Autos steigt. Auch im seelischen Bereich gibt es möglicherweise

\footnotetext{
${ }^{1}$ International Statistical Classification of Diseases and Related Health Problems.
} 
schreckliche Ereignisse, die auf irgendeine wunderbare Weise nicht zwangsläufig zu einer schweren seelischen Störung geführt haben. Manches Mal werden sie sogar im Gegenteil zum Ausgangspunkt einer neuen seelischen Architektur. Auch diese Beobachtung scheint wesentlicher Ausgangspunkt für psychoanalytische Betrachtungen zum Trauma zu sein.

Unter den verletzten Strukturen scheinen das ,gute innere Objekt“, die Integrität der Ich-Selbstregulation und die Grenze zwischen Selbst und Objekt von zentraler Bedeutung. Die Beschädigung dieser zentralen seelischen Strukturen und Funktionen wiederum könnte sich resultierend in einem Verlust des „Zartgefühls“ bemerkbar machen, um in dieser Überlegung beispielhaft eine traumapathologische Linie auszuziehen.

Auch diese Perspektive auf die verletzte Struktur ist bei Freud angelegt, wenn er zum Abschluss der Geschichte von „Katharina“ schreibt: „Ich hoffe, die Aussprache mit mir hat dem in seinem sexuellen Empfinden [kursiv U.L.] so frühzeitig verletzten Mädchen in etwas wohlgetan“ (1895, S. 195).

Immer wieder gibt es Traumen und Traumatisierungen. Da diese vor allem von Menschen ausgehen, geht die Geschichte vom Trauma wohl nie zu Ende.

Ulrich Lamparter

\section{Literatur}

Freud S (1895) Studien über Hysterie: Katharina. Gesammelte Werke, Bd 1, 5. Aufl. Fischer, Frankfurt a. M., S 184-193 (1977)

Nedelmann C (Hrsg) (2011) Phantasie und Realität. Kohlhammer, Stuttgart 